1918-9, for example, killed an estimated 40-50 million people. Research into influenza, in relation to its catastrophic potential, is underfunded. ${ }^{2}$ Worse still, we do not even seem able to provide sufficient vaccine to control epidemic influenza in the most developed countries. Little confidence exists that we could undertake a global immunisation programme for an influenza pandemic even if we had sufficient warning of its antigenic profile unless we can sharpen up on manufacture and distribution. Other notable examples of neglected diseases afflicting the world include antibacterial drug resistance, chronic obstructive pulmonary disease, alcoholic liver disease, and stroke. ${ }^{1}$

Secondly, the pharmaceutical industry is clearly unable to meet the needs of people with neglected diseases. This is not a criticism but a fact, for the pharmaceutical industry is a business and needs to provide its shareholders with a return on their investment. Many neglected diseases are unlikely to to do this and investment represents a great commercial risk. The industry will continue to play a major part in the discovery and development of drugs; but we need much greater pluralism in both the funding and discovery of novel treatments.

Examples of this pluralistic approach already exist. Substantial public funds for basic and clinical research, ultimately aimed at producing new forms of treatment, are available in the United States and to a lesser extent in Europe. The research agenda in the WHO report should be a major feature of the European Union's next research programme. For this we must rely on the influence of the Dutch presidency. But Europe also needs to harness the potential of public-private partnerships along the lines of the Global Alliance for TB Drug Development, or the Hereditary Disease Foundation in the United States. ${ }^{3}$ Public-private partnerships involve joint investment of resources by bodies including universities, government supported research organisations, pharmaceutical companies, venture capitalists, and research based charities. The Hereditary Disease Foundation's public-private partnerships include, for example, not only the foundation itself but several pharmaceutical companies and academic researchers of the Huntington Study Group collectively seeking an effective treatment for Huntington's disease; and that of the Global Alliance for TB Drug Development is a joint initiative between the Rockefeller Foundation, the Bill and Melinda Gates Foundation, $\mathrm{WHO}$, a group of university based investigators, and several private companies. ${ }^{3}$

Thirdly, the cost of developing a new drug must be reduced. ${ }^{4}$ Unless savage cuts can be made in the current cost of bringing a new drug to the market-over $\$ 800 \mathrm{~m}(£ 423 \mathrm{~m} ; € 605 \mathrm{~m})$ and rising at the rate of $10 \%$ a year ${ }^{5}-$ drug development will come to a standstill. Half this sum seems to be consumed by clinical development programmes. But real inroads need to be made into reducing all regulatory requirements if affordable new drugs are to be available to consumers. ${ }^{4}$ Promisingly, the problem has been acknowledged by both the US Food and Drug Administration and the European Medicines Agency, ${ }^{67}$ but international action is needed if these difficulties are to be resolved. Radical regulatory reform that is acceptable to the United States, the European Union, and Japan will be essential to relieve our current woes. This will have to include reducing the burdens of preclinical studies and adopting innovative approaches to investigating efficacy and safety in patients. ${ }^{4}$

Finally, the boundaries between primary and secondary prevention of disease clearly need to be redrawn. The silos currently occupied on the one hand by public health specialists and on the other by clinicians must be eroded. Prevention and control of disease need to go hand in hand. The transfer of the public health functions of the Health Development Agency to the National Institute for Clinical Excellence (NICE) as of April 2005 offers a real opportunity for this to happen-at least in England. ${ }^{8}$

\section{Michael D Rawlins professor}

Wolfson Unit of Clinical Pharmacology, Medical School, University of Newcastle, Newcastle upon Tyne NE2 4HH

(m.d.rawlins@ncl.ac.uk)

Competing interests: MR has been chairman of NICE since its formation in 1999

Kaplan W, Laing R. Priority medicines for Europe and the world. Geneva: World Health Organization, 2004. http://mednet3.who.int/prioritymeds report/index.htm (accessed 5 Nov 2004).

Treanor J. Weathering the influenza vaccine crisis. N Engl J Med 2004; $351 \cdot 2037-40$.

3 Moses H, Braunwald E, Martin JB, Their SO. Collaborating with industry-choices for the academic medical centre. $N$ Engl J Med 2002; industry-choi $1371-5$.

4 Rawlins MD. Cutting the cost of drug development. Nature Rev Drug Discov 2004;3:360-4.

5 DiMasi JA, Hansen RW, Grabowski HG. The price of innovation: new estimates of drug development costs.J Health Econ 2003;22:151-85.

6 European Medicines Agency. The European Medicines Agency road map to 2010: preparing the ground for the future. London: European Agency for the Evaluation of Medicinal Products, 2004

7 Food and Drugs Administration. Challenge and opportunity on the critical path to new medical products. Department of Health and Human Services: path to nedical pou html (accessed 6th Nov).

Department of Health. Report on the reconfiguration of the Departmen of Health's arm's length bodies. London: Department of Health, 2004 www.dh.gov.uk/PublicationsAndStatistics/Publications/Publication PolicyAndGuidance/PublicationsPolicyAndGuidanceArticle/fs/ en?CONTENT_ID = 4086081\&chk=y4UIfP (accessed 6 Nov 2004).

\title{
The treatment of lupus nephritis
}

\section{New and more conservative approaches in treatment are a major advance}

$\mathrm{F}$ or 30 years the US National Institutes of Health have dominated the treatment of lupus nephritis with controlled trials of monthly high dose intravenous pulse cyclophosphamide, now the standard treatment for nephritis and severe lupus. However, adverse effects such as ovarian failure and infections are significant with prolonged treatment. ${ }^{1}$ As most lupus patients are women of childbearing age, this price has been high and patients and clinicians are questioning this protocol. Recent studies offer two different approaches that may be as effective and better tolerated.

Additional references w1-w4 are on bmj.com 
The use of low dose cyclophosphamide, pioneered at St Thomas' Hospital, London, was recently compared with the US regimen in a European study. ${ }^{2}$ There were similar improvements in renal variables in proliferative lupus nephritis with both regimens but with a tendency to lower toxicity in the group receiving cyclophosphamide at low dosages. This probably reflects the shorter exposure to cyclophosphamide (three months $v 1$ year) and the early switch to azathioprine though longer term follow-up is needed. Similar results were shown in the European systemic vasculitis study, reflecting the trend towards shorter induction periods with cyclophosphamide. ${ }^{4}$

A different approach with mycophenolate mofetil is being widely studied. A randomised trial of mycophenolate mofetil versus oral cyclophosphamide in lupus nephritis ${ }^{5}$ was followed by case series, showing that it is well tolerated. A randomised trial in lupus nephritis showed advantages in efficacy and toxicity for azathioprine and mycophenolate mofetil over the National Institutes of Health's cyclophosphamide regimen as maintenance treatment. ${ }^{6}$ A further abstract shows that mycophenolate mofetil is as useful as the National Institutes of Health's regimen in induction but with less toxicity. ${ }^{7}$ These findings are prompting industry sponsored studies aimed at licensing mycophenolate mofetil for both induction and maintenance treatment. The National Institutes of Health's prolonged cyclophosphamide regimen has been useful, but its use may be drawing to a close.

Intravenous immunoglobulin is widely used in immunoglobulin deficiencies, Guillain-Barré syndrome, Kawasaki's disease, and systemic vasculitis. Numerous reports describe its use especially in patients with severe lupus complicated by sepsis, in whom it can be life saving. Although theoretical risks of infection (for example, with prion transmission) exist, these remain low compared with its efficacy. Randomised studies of intravenous immunoglobulin in lupus do not exist and it remains expensive.

A more targeted approach was pioneered by the team at University College Hospital London, who first used the B cell depleting anti-CD20 monoclonal antibody rituximab in rheumatoid arthritis. Some 20 lupus patients have now been treated, and the results are remarkable. Following two infusions of rituximab and intravenous cyclophosphamide, prolonged remissions have resulted in patients previously unresponsive to other treatments. ${ }^{8}$

Probably the most challenging aspect of lupus is central nervous system involvement. Recently, many central nervous system manifestations have been associated with antiphospholipid antibodies and this has altered our approach. For example, strokes, seizures, headache, and cognitive dysfunction, once thought to be due to inflammatory disease in lupus, are now clearly associated with the antiphospholipid (Hughes) syndrome. ${ }^{9 \mathrm{w}^{\mathrm{w} 2}}$ In patients with lupus who are positive for antiphospholipid antibodies, anticoagulation rather than immunosuppression needs to be considered. A difficult area is the overlap with multiple sclerosis. Demyelinating syndromes, although rare in lupus, may be associated with antiphospholipid antibodies. Similar appearances owing to antiphospholipid antibodies on magnetic resonance imaging may be impossible to distinguish from multiple sclerosis, leading to diagnostic confusion and uncertainty over treatment. ${ }^{10 w^{3}}$ Preliminary reports indicate benefit from anticoagulation.

The ramifications of antiphospholipid antibodies extend beyond the nervous system. For example, hypertension is a common complication of lupus nephritis, predictive of renal impairment. Recent studies show that stenosis of the renal artery and thrombotic microangiopathy associated with antiphospholipid antibodies may contribute to the development of hypertension and renal impairment in the context of lupus nephritis. This has led to the concept that anticoagulation may improve blood pressure control and prevent irreversible renal damage. ${ }^{11 \mathrm{w} 4}$

Women with lupus, especially with previous nephritis, were strongly advised to avoid pregnancy. This advice has changed dramatically, with the development of multidisciplinary clinics for lupus in pregnancy. Lupus nephritis considerably increases pregnancy related maternal and fetal morbidity risks but these risks can now be managed, providing the nephritis is quiescent at conception. ${ }^{12}$

Lupus was once considered a rare disease for which high dose corticosteroids were the only treatment. Arguably, a major advance in treatment has come from the widespread acceptance of a more conservative approach to treatment-especially with lower doses of corticosteroids. Clinicians in many specialties need to consider how best to manage patients with lupus, given the recent advances in treatment.

\section{David P D'Cruz consultant rheumatologist}

Graham R V Hughes consultant rheumatologist

Lupus Research Unit, Rayne Institute, St Thomas' Hospital, London SE17EH (david.d'cruz@kcl.ac.uk)

Competing interests: DDC and GRVH are participating in clinical trials of mycophenolate mofetil in lupus and are on the advisory board of Aspreva Pharmaceuticals.

1 Raptopoulou A, Sidiropoulos P, Boumpas D. Ovarian failure and strategies for fertility preservation in patients with systemic lupus erythematogies for fertility preservation
sus. Lupus 2004:13:887-90.

2 D'Cruz D, Cuadrado MJ, Mujic F, Tungekar MF, Taub N, Lloyd M, Khamashta MA, Hughes GRV. Immunosuppressive therapy in lupus nephritis. Clin Exp Rheumatol 1997;15: 275-82.

3 Houssiau FA, Vasconcelos C, D'Cruz D, Sebastiani GD, Garrido Ed Ede R, Danieli MG, et al. Immunosuppressive therapy in lupus nephritis: the Euro-lupus nephritis trial, a randomized trial of low-dose versus high-dose intravenous cyclophosphamide. Arthritis Rheum 2002;46: 2121-31.

4 Jayne D, Rasmussen N, Andrassy K, Bacon P, Tervaert JW, Dadoniene J, et al. European Vasculitis Study Group. A randomized trial of maintenance therapy for vasculitis associated with antineutrophil maintenance therapy for vasculitis associated with a
cytoplasmic autoantibodies. N Engl J Med 2003;349:36-44.

5 Chan TM, Li FK, Tang CS, Wong RW, Fang GX, Ji YL, et al. Efficacy of Chan TM, Li FK, Tang CS, Wong RW, Fang GX, Ji YL, et al. Efficacy of
mycophenolate mofetil in patients with diffuse proliferative lupus nephritis. Hong Kong-Guangzhou Nephrology Study Group. $N$ Engl J Med 2000;343:1156-62.

6 Contreras G, Pardo V, Leclercq B, Lenz O, Tozman E, O'Nan P, et al. Sequential therapies for proliferative lupus nephritis. $N$ Engl J Med 2004;350:971-80.

7 Ginzler E, Aranow C, Buyon J, Dooley MA, Merrill JT, Petri M, et al. A multicenter study of mycophenolate mofetil vs intravenous cyclophosphamide as induction therapy for severe lupus nephritis: preliminary results. Arthritis Rheum 2003;48(suppl):s647.

8 Leandro MJ, Edwards JC, Cambridge G, Ehrenstein MR, Isenberg DA. An open study of B lymphocyte depletion in systemic lupus erythematosus. Arthritis Rheum 2002;46:2673-7.

9 Sanna G, Bertolaccini ML, Cuadrado MJ, Laing H, Khamashta MA, Mathieu A, et al. Neuropsychiatric manifestations in systemic lupus erythematosus: prevalence and association with antiphospholipid antibodies. J Rheumatol 2003;30:985-92.

10 Cuadrado MJ, Khamashta MA, Ballesteros A, Godfrey T, Simon MJ, Hughes GR. Can neurologic manifestations of Hughes (antiphospholipid) syndrome be distinguished from multiple sclerosis? Analysis of 27 patients and review of the literature. Medicine (Baltimore). 2000;79:57-68.

11 Sangle SR, D'Cruz DP, Jan W, Karim MY Khamashta MA, Abbs IC Renal artery stenosis in the antiphospholipid (Hughes) syndrome and Renal artery stenosis in the antiphospholipid (H
hypertension. Ann Rheum Dis 2003;62:999-1002.

12 Moroni G, Ponticelli C. The risk of pregnancy in patients with lupus nephritis.J Nephrol 2003;16:161-7. 